\title{
Escala de avaliação da fadiga: Adaptação para profissionais da saúde
}

Fatigue assessment scale: Adaptation for health professionals Escala de evaluación de la fatiga: Adaptación para profesionales de la salud

\author{
Valdiney Veloso GOUVEIA ${ }^{1, a}$ \\ Gislene Farias de OLIVEIRA ${ }^{\text {b }}$ \\ Luís Augusto de Carvalho MENDESc \\ Luana Elayne Cunha de SOUZA ${ }^{\mathrm{d}}$ \\ Thiago Medeiros CAVALCANTI ${ }^{a}$ \\ Rômulo Lustosa Pimenteira de $\mathrm{MELO}^{\mathrm{a}}$ \\ Universidade Federal da Paraíba, João Pessoa, PB, Brasil ${ }^{a}$, Universidade Regional do Cariri, Crato, CE, Brasil ${ }^{\mathrm{b}}$, \\ Faculdade Maurício de Nassau, João Pessoa, PB, Brasilc, Universidade de Fortaleza, Fortaleza, CE, Brasil ${ }^{\mathrm{d}}$
}

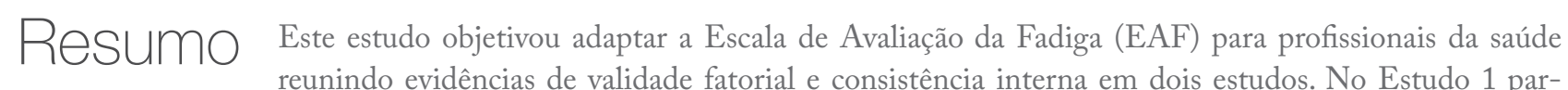
ticiparam 200 estudantes de três cursos: enfermagem $(n=56)$, medicina $(n=69)$ e psicologia $(n=75)$, com idades variando entre 18 e 39 anos $(M=22,0, D P=3,44)$, a maioria do sexo feminino $(64,5 \%)$ e solteira (90\%). Todos os estudantes responderam à EAF e a perguntas demográficas. A análise de componentes principais $(\mathrm{CP})$ revelou que a escala possui um componente, explicando $38,7 \%$ da variância total; alfa de Cronbach ( $\alpha$ ) foi 0,80 e índices de escalabilidade de $0,33(H)$ e 0,82 (Rho). Do Estudo 2 participaram 246 profissionais da saúde. A análise CP confirmou estrutura unidimensional, explicando $43,8 \%$ da variância total ( $\alpha=0,85, H=0,40$ e $R h o=0,87$ ), sendo 0,99 o coeficiente de congruência das soluções fatoriais (Estudos 1 e 2). Conclui-se que a medida apresenta evidências psicométricas adequadas de validade e precisão.

Palavras-chave:

Fadiga; saúde; avaliação.

\section{Abstract}

This study aimed to adapt the Fatigue Assessment Scale (FAS) to health professionals, combining evidence of factorial validity and reliability from two studies. In Study 1, the participants were 200 undergraduate students from three disciplines [Nursing $(n=56)$, Medicine $(n=69)$, and Psychology $(n=75)$ ], with ages ranging from 18 to 39 years $(M=22.0, S D=3.44)$, mostly female $(64.5 \%)$, and single $(90 \%)$, who answered the $F A S$ and demographic questions. Principal Component (PC) analysis revealed one component, explaining $38.7 \%$ of the total variance; Cronbach's alpha $(\alpha)$ was 0.80 and scalability indexes were $0.33(H)$ and $0.82(R h o)$. In Study 2, the participants were 246 health professionals. The PC analysis confirmed the one-dimensional structure, explaining $43.8 \%$ of the total variance $(\alpha=0.85, H=0.40$, and $R h o=0.87)$. The congruence coefficient was of 0.99 for the one-factor solution. In conclusion, the $F A S$ showed adequate psychometric evidence of validity and reliability.

Keywords:

Fatigue; health; assessment.

\section{Resumen}

Este estudio tuvo como objetivo adaptar la Escala de Evaluación de la Fatiga (EEF) para profesionales de la salud, reuniendo evidencias de su validez factorial y consistencia interna en dos estudios. En el Estudio 1 participaron 200 estudiantes, de enfermería $(n=56)$, medicina $(n=69)$ y psicología $(n=75)$, con edades entre 18 y 39 años $(M=22.0, D S=3.44)$, la mayoría mujeres $(64.5 \%)$ y solteras (90\%). Todos los estudiantes contestaron a la $E E F$ y a preguntas demográficas. El análisis de componentes principales (ACP) reveló un componente general, explicando el $38.7 \%$ de la varianza total; su alfa de Cronbach $(\alpha)$ fue de 0.80 e índices de

\footnotetext{
Endereço para correspondência: Universidade Federal da Paraíba, Centro de Ciências Humanas Letras e Artes - Campus I, Departamento de Psicologia, Cidade Universitária, s/n, João Pessoa, PB, Brasil 58051-900. E-mail: vvgouveia@gmail.com
} 
escalabilidad de 0.33 (H) y 0.82 ( $R$ ho). En el Estudio 2 los participantes han sido 246 profesionales de la salud. El ACP confirmó la estructura unidimensional, explicando el $43.8 \%$ de la varianza total $(\alpha=0.85, H=0.40$ y $R$ ho $=0.87$ ), siendo el coeficiente de congruencia de las soluciones factoriales (Estudios 1 y 2) de 0.99. Por fin, se ha concluido que esta medida presenta evidencias psicométricas adecuadas de validez y confiabilidad.

Palabras-clave:

Fatiga; salud; evaluación.

1

fadiga é uma queixa comum entre pessoas da população geral e pacientes clínicos (Cella \& Chalder, 2010; Fritschi \& Quinn, 2010; Michielsen, De Vries, Drent, \& Peros-Golubicic, 2005; Michielsen, Peros-Golubicic, Drent, \& De Vries, 2006), mas, sobretudo, entre trabalhadores de diversas ocupações (Linsey, Barker, \& Nussbaum, 2011; Moore, Gupta, \& Duval Neto, 2013; Oliveira, Viganó, Lunardelli, Canêo, \& Goulart, 2010; Vasconcelos, Fischer, Reis, \& Moreno, 2011; Wilson, 2013), e é possível defini-la a partir de diferentes abordagens. Por exemplo, de uma perspectiva geral, a fadiga compreende uma sensação subjetiva de cansaço com componentes comportamentais, emocionais e cognitivos, sendo uma sintomatologia de várias doenças, como câncer, esclerose múltipla ou doença de Parkinson (Mota, Pimenta, \& Caponero, 2012; Sanches \& Cardoso, 2012), refletindo-se em baixa atividade física e sonolência (Oliveira et al., 2010). $\mathrm{Na}$ perspectiva do trabalho, ela pode ser definida como uma experiência de cansaço, desgosto pela atividade atual e falta de vontade para continuar (Kant et al., 2003). Assim, como consequência, há comprometimento da competência e menor disposição para desenvolver ou manter objetivos (De Vries, Michielsen, \& Van Heck, 2003).

Vale salientar que a fadiga não é necessariamente um sintoma de uma determinada doença, mas pode ser entendida como uma experiência diária de desgaste, que afeta diversas pessoas da população (Yang \& Wu, 2005). De fato, ela pode ser compreendida não como um efeito adverso, mas como uma adaptação psicológica ou um mecanismo de defesa empreendido pelas pessoas para fazer frente ao risco de esforço exagerado ou exaustão (Van Dijk \& Swaen, 2003). Partindo dessa perspectiva, diversos estudos têm abordado a fadiga, relacionando-a com variáveis como depressão (De Vries, Van der Steeg, \& Roukema, 2009), baixa qualidade de vida (Sanches \& Cardoso, 2012) e traços de personalidade, a exemplo de extroversão (De Vries et al., 2009).

Devido a sua relevância, criou-se um programa de pesquisa (Fatigue at Work) a fim de conhecer a etiologia, o curso e as consequências para as pessoas em estados agudos e prolongados de fadiga (Andrea, Kant, Beurskens, Metsemakers, \& Van Schayck, 2003; Janssen, Kant, Swaen, Janssen, \& Schröer, 2003; Van Dijk \& Sawen, 2003). Com efeito, é precisamente no âmbito do trabalho que essa sintomatologia se faz mais preponderante, estando correlacionada a diversas variáveis laborais, carecendo de atenção especial.

\section{Fadiga no Contexto do Trabalho}

Diversas pesquisas têm demonstrado que a fadiga no trabalho pode causar diversos problemas no ambiente laboral, como o comprometimento das habilidades do indivíduo e de seu desempenho na função assumida (Oliveira et al., 2010). O turno de trabalho é outro fator estreitamente relacionado à presença de fadiga, o que foi comprovado em estudos prévios (Janssen et al., 2003), cujos autores observaram maior presença de fadiga entre trabalhadores com turnos diferentes, que trabalham em horários quebrados, do que entre os que atuam em turnos regulares. Desse modo, a fadiga foi mencionada neste estudo como a maior causa de intolerância à variação dos turnos de trabalho.

Alguns autores têm observado que, quando a fadiga tem um tempo relativamente longo de duração, pode levar a doenças leves e deficiência no trabalho. Michielsen, Willemsen, Croon, De Vries e Van Heck (2004a) constataram que a carga de trabalho e a percepção de estresse são tidas como fortes preditoras de fadiga geral e exaustão emocional. Nessa direção, Janssen et al. (2003) abordaram a fadiga como um importante preditor de ausência no trabalho, que pode ser vista como um mecanismo de enfrentamento, um sintoma de estresse e de doença ou, ainda, uma reação à percepção desses sintomas.

A fadiga também pode ser vista como um fator de risco para acidentes no trabalho. De modo concreto, estima-se que ela influencie na etiologia dos acidentes de trabalho por dois motivos: (a) pode diminuir a habilidade para processar informações sobre situações perigosas e (b) pode diminuir a habilidade para responder adequadamente a tais situações (Van Dijk \& Sawen, 2003). Essas são razões suficientes para a procura de um psicólogo ou médico por aqueles que enfrentam indícios de fadiga, tendo sido igualmente observado em estudo

Rev. Psicol., Organ. Trab., jul-set 2015, vol. 15 num. 3 
desenvolvido por Andrea et al. (2003). Tais autores examinaram a associação entre atribuições de fadiga, nível da doença, problemas de saúde física e de saúde mental e características psicossociais do trabalho. De forma específica, observaram que a fadiga é uma queixa comum entre trabalhadores que procuram um clínico geral, e que atribuem sua fadiga a problemas somáticos e psicológicos. Do mesmo modo, constatou-se que tais trabalhadores não somente apresentaram níveis elevados de fadiga, assim como uma percepção negativa de seu ambiente de trabalho.

Os fatores listados anteriormente parecem exercer forte influência no recebimento de futuros benefícios por conta de doença ou deficiência no trabalho. E, de fato, alguns têm relação direta com o recebimento de benefícios, como a diminuição do desempenho e da habilidade no cargo exercido e o surgimento de doenças leves (Kant et al., 2003; Michielsen et al., 2004a). Considerando as informações anteriores e levando em conta as práticas médicas e psicológicas em contexto laboral, existem ao menos quatro razões que endossam o fato de a fadiga ser identificada como uma preditora de problemas de saúde dos trabalhadores (Janssen et al., 2003; Kant et al., 2003; Van Dijk \& Sawen, 2003): (a) a alta prevalência de casos de fadiga na população de trabalhadores, (b) o estado de fadiga ser particularmente persistente nesse grupo, (c) a fadiga ser uma condição incapacitante de trabalhadores e (d) uma proporção substancial de trabalhadores receber benefícios por deficiência ou doença pautada no diagnóstico de "reação exógena ou adaptativa", conforme a CID-10, sendo tal diagnóstico composto por características como estresse no trabalho, sobrecarga e burnout, das quais a fadiga é um sintoma integrante.

Desse modo, parece evidente ter em conta a fadiga como forma de entender suas implicações e, posteriormente, primar pela qualidade de vida e de trabalho dos indivíduos. No entanto, demanda-se avaliá-la previamente de maneira adequada no contexto laboral, contando com instrumentos curtos e que apresentem qualidades psicométricas aceitáveis. Esse é precisamente o foco deste artigo, requerendo-se, nesta oportunidade, tomar conhecimento das avaliações existentes dessa sintomatologia.

\section{Medindo a Fadiga}

Procurando conhecer o panorama sobre as medidas disponíveis a respeito da fadiga, Shahida, Shena e Shapiro (2010) realizaram uma revisão da literatura que relacionava 12 instrumentos, em que a variação de seu número de itens era de 9 (Fatigue Severity Scale; Krupp, LaRocca, Muir-Nash, \& Steinberg, 1989) a 50 (Shapiro et al., 2002), com média superior a 20 itens. Destes, 8 tinham como grupo de interesse pacientes com diversos problemas de saúde (p. ex., câncer, esclerose múltipla, fadiga crônica). Enquanto alguns autores definiram a fadiga como unifatorial (p. ex., Krupp et al., 1989), outros propuseram estruturas com dois (Lee, Hicks, \& Ninomurcia, 1991), quatro (Schwartz, Janford, \& Krupp, 1993) ou cinco fatores (Smets, Garssen, Bonk, \& de Haes, 1995). Coerentemente, todos apresentaram consistência interna (alfa de Cronbach) satisfatória, variando de 0,83 (Krupp et al., 1989) a 0,98 (Schwartz et al., 1993).

Partindo de medidas prévias sobre fadiga, De Vries, Michielsen, Van Heck e Drent (2004) criaram a Escala de Avaliação da Fadiga (EAF), instrumento que tem sido utilizado em estudos com população de pacientes com sarcoidose, dentre os quais o de Michielsen et al. (2006), corroborando a fadiga como uma queixa experimentada pela maioria deles. Os autores também observaram que a fadiga configura-se como uma importante preditora (peso negativo) de indicadores de qualidade de vida. É importante ressaltar que esse instrumento é parcimonioso, reúne 10 itens, e considera a fadiga como um construto unidimensional, apresentando consistência interna satisfatória. Além disso, conta com adaptação ao contexto brasileiro, reunindo evidências preliminares de seus parâmetros psicométricos, mas considerando apenas uma amostra de estudantes universitários (Oliveira, Gouveia, Peixoto, \& Soares, 2010).

Em resumo, existem diferentes medidas de fadiga, que apresentam estruturas fatoriais e coeficientes de consistência interna variados, a maioria delas dirigidas a grupos específicos de pessoas portadoras de alguma enfermidade, mas também há aqueles com pessoas da população geral. Diante desse contexto, considerando o fato de a EAF ser uma medida curta e que reúne parâmetros psicométricos satisfatórios, é pertinente considerar seu uso no contexto laboral, especificamente na área da saúde, permitindo rápida aplicação sem comprometer a qualidade na avaliação. Nesse sentido, objetiva-se com este estudo conhecer evidências de sua validade fatorial entre profissionais da saúde no Brasil que têm sido expostos a elevados níveis de estresse que causam distúrbios do sono e fadiga (Owens, 2012). Assim, demanda-se detalhar essa medida. 


\section{Escala de Avaliação da Fadiga}

Devido ao rápido crescimento do número de pessoas que sofrem de fadiga, o interesse por esse construto tem aumentado consideravelmente, o que tem levado a um intenso debate sobre sua conceituação, seus determinantes, suas manifestações e suas consequências (Michielsen, De Vries, \& Van Heck, 2003). Entretanto, no que se refere à medida em si, o maior debate tem sido quanto a sua dimensionalidade. Chalder et al. (1993), por exemplo, sugerem a presença de dois fatores (fadiga física e fadiga psicológica), mas também são comuns os modelos multifatoriais (Lee et al., 1991; Schwartz et al., 1993; Shapiro et al., 2002). Okuyama et al. (2000), por sua vez, identificaram três fatores para uma medida específica de fadiga em pacientes com câncer, enquanto Dagnelie et al. (2006) indicaram uma solução com quatro fatores para a Escala de Fadiga de Piper.

Destaca-se que, com frequência, os resultados que dão conta de estruturas bi e multifatoriais partem de análises pouco robustas, considerando para a definição do número de fatores, por exemplo, o critério de Kaiser, isto é, valor próprio igual ou superior a 1 . Sabe-se que esse critério superestima o número de fatores, fazendo a solução fatorial ser inflada (Tabachnick \& Fidell, 2013). Nesse sentido, discute-se a possibilidade de uma medida unidimensional, que conta com a vantagem de parcimônia, facilitando seu emprego em estudos epidemiológicos, naqueles de triagem ou quando há necessidade de incluir outras medidas para conhecer o perfil de determinado grupo ou população (Krupp et al., 1989).

Um instrumento que se apresenta como promissor é a EAF, pois reúne apenas 10 itens e possui propriedades psicométricas adequadas. Portanto, considerando a necessidade de contar com uma medida breve de fadiga, que reúna evidências de validade fatorial e consistência interna, este artigo tem como objetivo adaptá-la para o uso no contexto de profissionais de saúde, demandando-se, nesta oportunidade, descrevê-la de maneira mais detalhada. Proposta por Michielsen, De Vries, Van Heck, Van de Vijver e Sijtsma (2004b), a EAF mede fadiga física e fadiga psicológica, e foi elaborada a partir de dois estudos realizados na Holanda:

Estudo 1. Examinou-se a dimensionalidade de medidas de fadiga em uma amostra de trabalhadores cuja carga mínima de trabalho era de 20 horas semanais, e que concordaram em completar os questionários que compunham um estudo longitudinal. Participaram 452 homens com média de 41 anos (amplitude de 20 a 63) e 412 mulheres com média de 39 anos (amplitude de 18 a 65). Eles responderam a cinco instrumentos: o Checklist Individual Strength (CIS-20), a Emotional Exhaustion(EE), o Maslach Burnout Inventorye a subescala energy and fatigue, do World Health Organization Quality of Life (EF-WHOQOL), e a Fatigue Scale (FS). Foram feitas análises fatoriais exploratórias e uma Análise de Mokken para as escalas, individualmente, e o conjunto total de itens destas medidas.

Essas análises revelaram soluções unifatoriais de tais medidas, que foram corroboradas pelos respectivos scree plots (Critério de Catell) e, em razão da variância total explicada, variou de 40 (FS) a 69\% (EF-WHOQOL). $\mathrm{Na}$ interpretação da Análise de Mokken, constatou-se que, dos 40 itens, 37 formaram uma escala consistente ( $H=0,47$; Molenaar \& Sijtsma, 2000), e os alfas de Cronbach das escalas individuais foram satisfatórios, variando de 0,76 (FS-Mental Fatigue) a 0,94 (CIS-20). Portanto, sugeriu-se a unidimensionalidade da fadiga com uma nova medida, que fosse curta e de fácil administração.

Estudo 2. Procurou-se construir o novo instrumento de autorrelato de fadiga, conhecendo sua validade de conteúdo e precisão. Duas amostras compuseram este estudo; a do Estudo 1 e outra, com 1.128 homens, com idade média de 46 anos (amplitude de 16 a 87), e 765 mulheres, com idade média de 42 anos (amplitude de 16 a 87). Empregaram-se dois critérios para selecionar os itens: (a) excluir aqueles respondidos apenas por grupos específicos (p. ex., trabalhadores) e (b) agrupar os semanticamente equivalentes. Partindo desses critérios, nove grupos de itens foram formados: (1) sentir-se incomodado devido à fadiga; (2) sentir-se fisicamente cansado; (3) ficar cansado rapidamente; (4) nível de energia; (5) concentração; (6) incapacidade para pensar claramente; (7) quantidade de atividade diária; (8) problemas para começar a fazer algo; (9) não sentir vontade de fazer qualquer coisa.

Posteriormente, em cada grupo semântico, o item com maior saturação na solução fatorial dos 40 itens foi escolhido, resultando em um total de 9 itens e, por último, um item sobre fadiga mental foi incluído. Portanto, a versão experimental da EAF consistiu de 10 itens cuja análise fatorial indicou um único fator, explicando $48 \%$ da variância total. A Análise de Mokken revelou que esses itens conformam uma escala consistente $(H=$ 0,47), apresentando alfa de Cronbach de 0,87.

Em resumo, tais estudos indicaram que as escalas avaliadas corroboraram a ideia de que a fadiga se configura adequadamente como um construto unidimensional. Consoante com esse entendimento, Michielsen et 
al. (2003) checaram as propriedades psicométricas da EAF, como validade convergente com outras medidas de fadiga e validade divergente com depressão e estabilidade emocional, mostrando que tais construtos diferem da fadiga. Do mesmo modo, De Vries et al. (2003) desenvolveram um estudo e checaram a validade convergente da EAF, sugerindo que essa é uma medida promissora da fadiga.

Outros autores confirmaram evidências de sua validade fatorial e consistência interna em pacientes com sarcoidose (Michielsen et al., 2005), mulheres com doenças de mama (De Vries, Van der Steeg, \& Roukema, 2010), pacientes com esclerose múltipla, doença de Parkinson, e que sofreram acidente vascular cerebral (Elbers et al., 2012). Seu uso tem-se ampliado para pacientes com insuficiência cardíaca crônica (Smith et al., 2007) e mulheres em estado puerperal (Giallo, Wade, Cooklin, \& Rose, 2011).

Porfim, existem versões adaptadas para os contextos alemão(Hinz, Fleischer, Brähler, Wirtz, \&Bosse-Henck, 2011) e estadunidense (De Kleijn, Elfferich, \& De Vries, 2009), cujos parâmetros psicométricos foram na direção dos aqui descritos anteriormente. Portanto, em razão desses achados, parece pertinente o empreendimento de adaptar a escala para o contexto brasileiro, conhecendo evidências de sua validade fatorial e precisão em amostras de pessoas que têm atividades no contexto da saúde. Esses aspectos motivaram este artigo, cujos respectivos estudos serão descritos a seguir.

\section{Estudo 1. Escala de Avaliação da Fadiga: Estudantes da área da saúde}

Este primeiro estudo objetivou adaptar a EAF para o cenário brasileiro, reunindo as primeiras evidências de sua validade fatorial e consistência interna em contexto universitário da saúde. Pretendeu-se checar, principalmente, a pertinência de assumir sua estrutura unifatorial.

\section{MÉTODO}

\section{Participantes}

Participaram deste estudo 200 estudantes universitários de três cursos da área da saúde [enfermagem $(n=56)$, medicina $(n=69)$ e psicologia $(n=75)]$, com idades variando de 18 a 39 anos $(M=22,0, D P=3,44)$, a maioria do sexo feminino $(64,5 \%)$ e solteira $(90,0 \%)$.

\section{Instrumentos}

Os participantes responderam à EAF (Michielsen et al., 2004b) e a quatro perguntas demográficas: idade, sexo, estado civil e curso de graduação. Conforme descrito anteriormente, a EAF é composta por 10 itens (p. ex., Sinto-me incomodado devido à fadiga; Fico cansado muito rapidamente), respondidos em escala de cinco pontos, variando de 1 (Nunca) a 5 (Sempre), devendo o participante indicar como tem se sentido, de forma geral, nos últimos 30 dias. Informações acerca de seus parâmetros psicométricos foram apresentadas previamente, quando se detalhou sua elaboração.

Primeiramente, com o fim de traduzi-la do original, em inglês, para o português, efetuou-se o procedimento de tradução reversa (back translation). $\mathrm{Na}$ ocasião, contou-se com dois colaboradores bilíngues; um fez a tradução do inglês para o português e o outro traduziu esta versão em português novamente para o inglês. Tais versões foram comparadas a fim de verificar se havia equivalência entre elas, sem perda de conteúdo e de sentido dos itens ao traduzi-los. Depois, procedeu-se à sua validação semântica. Nessa oportunidade, participaram dez estudantes universitários do primeiro semestre de psicologia, com idade entre 18 e 57 anos, sendo igualmente distribuídos quanto ao sexo. Essa validação permitiu verificar se as instruções eram compreensíveis, se a escala de resposta utilizada era adequada e se os itens eram inteligíveis. Constatado que não foram necessárias modificações substanciais, chegou-se à versão brasileira dessa escala, que poderá ser obtida com os autores deste artigo.

\section{Procedimentos de coleta de dados e cuidados éticos}

Os participantes responderam aos instrumentos individualmente, em ambiente coletivo de sala de aula. Três colaboradores treinados ficaram responsáveis por sua aplicação e, após a autorização do professor da disciplina, eles se apresentavam e solicitavam a colaboração dos presentes a responderem voluntariamente aos questionários. Uma vez tendo concordado em participar, os estudantes preencheram um termo de consentimento livre e esclarecido. A todos foi enfatizado o caráter voluntário, anônimo e confidencial da participação, 
seguindo o que estabelecem os procedimentos éticos em vigor. Em média, 15 minutos foram suficientes para concluir sua participação.

\section{Procedimentos de análise de dados}

A análise dos dados foi realizada com o SPSS (Statistical Package for the Social Sciences) na versão 18. Procurando verificar a estrutura fatorial da escala, os dados foram submetidos a uma análise de CP. Nesse sentido, além dos critérios de Kaiser (valor próprio igual ou superior a 1) e Cattell (distribuição gráfica dos valores próprios), realizou-se uma análise paralela por ser considerada uma estratégia mais robusta para definir o número de componentes a serem extraídos. Depois de determinada a dimensionalidade da escala, calculouse o alfa de Cronbach $(\alpha)$ para o componente resultante.

A partir do Programa R (Team, 2012) foi realizada uma Análise Mokken, utilizando código aberto (open source), que fornece as mesmas análises da versão comercial do MSP (Molenaar \& Sijtsma, 2000). O propósito foi comprovar a unidimensionalidade da EAF. A MSA é uma análise não paramétrica, baseada na Teoria de Resposta ao Item (TRI), que busca verificar os pressupostos de homogeneidade monotônica e monotonicidade dupla. Por meio dela, busca-se comprovar, principalmente, os índices de escalabilidade $H$ (para a escala total) e $H s$ (para cada item), considerando-se aceitáveis valores acima de 0,30 , e o $R$ ho de Mokken, com valores ideais acima de 0,80 .

\section{RESULTADOS}

Tomando como base o $\operatorname{KMO}(0,83)$ e o Teste de Esfericidade de Bartlett $\left[\chi^{2}(45)=614,89, p<0,01\right]$, comprovou-se a adequação de fatorializar a matriz de correlações entre os itens da EAF, justificando realizar a análise fatorial. Nesse caso, decidiu-se efetuar uma análise de $\mathrm{CP}$, coerente com o que tem sido encontrado na literatura, fixando a extração apenas de um componente, que foi confirmado quando se observou a distribuição gráfica dos valores próprios (critério de Cattell). Contudo, não pôde ser corroborado pela análise paralela, considerando 1.000 simulações com 200 casos e 10 variáveis (parâmetros do banco de dados original) e, levando em conta os 5 primeiros valores próprios observados $(3,87 ; 1,49 ; 1,06 ; 0,73$; e 0,62$)$, percebe-se que os dois primeiros foram superiores aos valores próprios médios simulados $(1,37 ; 1,25 ; 1,16 ; 1,09$; e 1,02), sugerindo dois componentes.

Então, a análise Mokken foi empregada para dirimir dúvidas e, assim, foi comprovada a pertinência de admitir a unifatorialidade da medida avaliada, que apresentou índices de escalabilidade aceitáveis $(H=0,33$ e $R h o=0,82$ ). Porém, ressalta-se que o item 3 (Não faço muitas coisas durante o dia) apresentou índice abaixo do recomendado na literatura $(H s=0,09)$, e os demais itens tiveram coeficientes $H s$ entre 0,25 (Item 6) e 0,45 (Item 5), com valor médio de 0,33. Assim, decidiu-se admitir a estrutura unifatorial, sendo os resultados correspondentes apresentados na coluna estudantes da Tabela 1.

Conforme observado na tabela, todas as saturações foram satisfatórias, com valores superiores ao recomendado $(0,30)$, exceto para o item 3 , que apresentou saturação de 0,22 . Esse componente geral de fadiga resultou em valor próprio de 3,87, explicando $38,7 \%$ da variância total. O conjunto de itens apresentou um índice de consistência interna de 0,80 e homogeneidade (correlação média item-total) de 0,29, variando de -0,12 (entre os itens 10 [Posso me concentrar bem quando estou fazendo algo] e 3 [Não faço muitas coisas durante o dia]) a 0,61 (entre os itens 5 [Sinto-me exausto fisicamente] e 1 [Sinto-me incomodado devido à fadiga]). A consistência interna (alfa de Cronbach, $\alpha$ ) desse fator geral foi de 0,80 . 
TABELA 1. Análise de Componentes Principais da FAS em estudantes e profissionais

\begin{tabular}{|c|c|c|c|c|}
\hline \multirow{2}{*}{ Conteúdo dos itens } & \multicolumn{2}{|c|}{ Estudantes } & \multicolumn{2}{|c|}{ Profissionais } \\
\hline & $\lambda$ & $r_{i . t}$ & $\Lambda$ & $r_{i . t}$ \\
\hline 05. Sinto-me exausto fisicamente. & 0,78 & 0,40 & 0,78 & 0,44 \\
\hline 02. Fico cansado muito rapidamente. & 0,78 & 0,39 & 0,76 & 0,43 \\
\hline 09. Sinto-me exausto mentalmente. & 0,75 & 0,38 & 0,74 & 0,41 \\
\hline 01. Sinto-me incomodado devido à fadiga. & 0,70 & 0,35 & 0,71 & 0,41 \\
\hline 08. Não sinto vontade de fazer nada. & 0,68 & 0,33 & 0,69 & 0,38 \\
\hline 07. Tenho problemas em pensar claramente. & 0,58 & 0,29 & 0,65 & 0,36 \\
\hline 04. Tenho suficiente energia para o meu dia a dia.* & 0,58 & 0,27 & 0,64 & 0,37 \\
\hline 10. Posso me concentrar bem quando estou fazendo algo.* & 0,48 & 0,25 & 0,64 & 0,36 \\
\hline 06. Tenho problemas para começar coisas. & 0,46 & 0,22 & 0,52 & 0,28 \\
\hline 03. Não faço muitas coisas durante o dia. & 0,22 & 0,09 & 0,38 & 0,21 \\
\hline Número de itens & 10 & & 10 & \\
\hline Valor Próprio & 3,87 & & 4,38 & \\
\hline \% de variância explicada & 38,70 & & 43,76 & \\
\hline alfa de Cronbach & 0,80 & & 0,85 & \\
\hline
\end{tabular}

Nota. * Item invertido. $\lambda=$ Saturação (carga fatorial) e $r_{i . t}=$ Correlação item-total.

\section{DISCUSSÃO PARCIAL}

Este estudo reuniu evidências da unifatorialidade da EAF, considerando múltiplos critérios, como Kaiser, Cattell e Horn. Este último (análise paralela), diferentemente dos anteriores, indicou uma estrutura bifatorial. Entretanto, visando resolver qualquer dúvida, comprovaram-se a homogeneidade monotônica e a monotonicidade por meio da Análise Mokken, que apresentou índice de escalabilidade $H$ de Löevinger adequado. Assim, reforçou-se a estrutura unifatorial desta escala, e a consistência interna (alfa de Cronbach, $\alpha$ ) e a homogeneidade (correlação média item-total) endossaram sua unidimensionalidade. No entanto, apenas estudantes universitários colaboraram com o presente estudo, demandando conhecer como essa medida funciona entre profissionais da saúde.

\section{Estudo 2. Escala de Avaliação da Fadiga: Profissionais da área da saúde}

O segundo estudo é uma tentativa de replicar o descrito anteriormente, procurando conhecer evidências de validade fatorial e consistência interna da EAF no contexto de profissionais da saúde. Procura-se, ainda, avaliar a congruência entre a estrutura fatorial resultante comparada com a do Estudo 1.

\section{MÉTODO}

\section{Participantes}

Participaram deste estudo 246 profissionais (67 enfermeiros, 81 médicos e 98 psicólogos) com idades variando de 19 a 76 anos $(M=42,8, D P=11,53)$, a maioria do sexo feminino (81,2\%) e casada $(59,6 \%)$. Quanto à situação laboral, $86 \%$ indicaram estar empregados, exercendo uma (43\%) ou duas $(84,2 \%)$ atividades, trabalhando em média 40 horas semanais. Dos profissionais de psicologia, apenas 30,2\% trabalham na área hospitalar. As porcentagens correspondentes foram maiores para médicos $(67,9 \%) \mathrm{e}$, principalmente, para enfermeiros $(76,1 \%)$.

\section{Instrumentos}

Os participantes responderam ao mesmo questionário descrito no Estudo 1, formado pela EAF e pela caracterização demográfica. Nesse caso, mantiveram-se as variáveis idade, sexo e estado civil, mudando o curso de graduação para a área de especialidade em que era formado, acrescentando setor ocupacional, situação laboral e carga horária semanal. 


\section{Procedimentos de coleta de dados e cuidados éticos}

Os profissionais foram localizados a partir de seus respectivos conselhos profissionais, e a coleta de dados foi realizada de duas formas: por meio de correspondência e de entrega pessoal de questionários. Em seguida, um envelope foi encaminhado ao endereço cadastrado de cada profissional selecionado aleatoriamente, que continha: (a) o questionário; (b) informações sobre a importância e a finalidade do estudo, bem como a maneira de respondê-lo; (c) uma solicitação de preenchimento e procedimento para devolução dos questionários; e (d) um envelope selado e sobrescrito para devolução, sem identificação do remetente. Os instrumentos eram autoaplicáveis, devendo os participantes lê-los e respondê-los individualmente, em situação de anonimato, e as respostas foram dadas sem determinação de tempo. No caso dos profissionais que receberam os questionários pessoalmente, estes também foram selecionados aleatoriamente, sendo contatados no endereço de registro em seu conselho profissional. Foi agendado com cada um destes o dia em que seria feita a devolução dos questionários preenchidos, que seriam colocados em um envelope lacrado, sem qualquer identificação, e depositado em uma urna. Seguiram-se os procedimentos de pesquisa com seres humanos, de acordo com o que determina a legislação vigente.

\section{Procedimentos de análise de dados}

Foram realizadas as mesmas análises descritas no Estudo 1, porém incluiu-se o teste de congruência entre soluções fatoriais, calculado com o programa Excel a partir da seguinte fórmula: $r_{\text {congruenencia }}=\left(\sum \mathrm{ab}\right) /\left[\left(\sum \mathrm{a}^{2}\right) \cdot\left(\sum\right.\right.$ $\left.\left.\mathrm{b}^{2}\right)\right]^{1 / 2}$, onde "a" e "b" correspondem às saturações dos componentes que estão sendo comparados. Essa fórmula permite avaliar se as soluções fatoriais encontradas nesse estudo são similares àquelas descritas no Estudo 1 , sendo a constatação de uma estrutura unifatorial; valores de $r_{\text {congruência }}$ iguais ou superiores a 0,90 são uma evidência da congruência das soluções fatoriais (Reynolds \& Ramsey, 2003).

\section{RESULTADOS}

Tomando em conta o KMO $(0,86)$ e o Teste de Esfericidade de Bartlett $\left[\chi^{2}(45)=865,92, p<0,01\right]$, pareceu pertinente proceder à análise fatorial, então, decidiu-se realizar uma análise $\mathrm{CP}$, fixando a extração de um componente e, considerando o critério de Cattell, pareceu clara a existência de um componente geral de fadiga. Esse resultado foi corroborado com a análise paralela, admitindo 1.000 simulações com 246 casos e 10 itens e, levando em conta os 5 primeiros valores próprios observados $(4,38 ; 1,14 ; 0,96 ; 0,80$; e 0,65$)$, constatou-se que o primeiro foi superior aos simulados $(1,32 ; 1,22 ; 1,15 ; 1,08 ;$ e 1,02$)$, reforçando a pertinência de extrair apenas um componente. A análise Mokken ofereceu evidências na mesma direção, isto é, verificou-se que apenas o item 3 apresentou índice abaixo do recomendado $(H s=0,23)$, tendo os demais itens variado de 0,30 (Item 10) a 0,45 (Item 8), com média de 0,40 , com os indicadores aceitáveis de escalabilidade da medida $(H=0,40$ e $R$ ho $=0,86)$.

Os resultados da análise $\mathrm{CP}$, fixando a extração de um componente geral, podem ser observados na coluna profissionais da Tabela 1, que mostra que todas as cargas fatoriais foram superiores a 0,30, variando de 0,38 (Item 3 - Não faço muitas coisas durante o dia) a 0,78 (Item 5 - Sinto-me exausto fisicamente). Esse componente único explicou $43,8 \%$ da variância total, tendo apresentado $\alpha=0,85$ e homogeneidade de 0,36 , variando de 0,07 (entre os itens 3 e 10, como no Estudo 1) a 0,60 (entre os itens 1 [Sinto-me incomodado devido à fadiga] e 2 [Fico cansado muito rapidamente]). Por último, comparando as saturações deste estudo com aquelas do estudo prévio (Tabela 1), observou-se um coeficiente de congruência de 0,99. Portanto, parece plausível admitir uma estrutura unifatorial comum para a medida de fadiga considerada.

\section{DISCUSSÃO PARCIAL}

Esse segundo estudo reuniu evidências adicionais de adequação psicométrica da EAF. Os três critérios (Kaiser, Cattell e Horn) indicaram a extração de um fator geral cuja estrutura unidimensional foi corroborada em razão do índice de escalabilidade, e a consistência interna e a homogeneidade dessa medida apoiaram considerá-la como medindo um fator. Por fim, a convergência dos achados descritos nos dois estudos, refletido no índice de congruência da estrutura unifatorial, reforça a adequação da EAF. 


\section{DISCUSSÃO GERAL}

O objetivo principal deste estudo foi adaptar a EAF para o uso com profissionais da saúde, reunindo evidências de sua validade fatorial e consistência interna. Estima-se que este tenha sido alcançado. Entretanto, admite-se limitações potenciais dos estudos, como o fato de as amostras não terem sido aleatórias e contarem apenas com estudantes, médicos, enfermeiros e psicólogos. Assim, não se pode pretender generalização para além desse escopo, ficando evidente a necessidade de futuros estudos que contem com profissionais de outras especialidades e regiões do país. Contudo, ressalta-se a natureza psicométrica dos estudos, cujas amostras foram suficientes para as análises efetuadas.

Destacou-se a importância de conhecer a fadiga em razão de suas consequências na diminuição do comprometimento e na menor disposição para desenvolver ou manter objetivos diretos (Oliveira et al., 2010). Nesse sentido, este artigo oferece importante contribuição à temática, incentivando que se avalie objetivamente a fadiga de trabalhadores. A facilidade de manuseio dessa medida pode ser algo bem favorável, considerando a escala de resposta e o número reduzido de itens. Ressalta-se que instrumentos breves são bem aceitos, principalmente quando a intenção for utilizá-los junto a outros instrumentos, diminuindo o cansaço do respondente ou mesmo a desistência da participação da pesquisa.

Quanto à estrutura fatorial, ambos os estudos indicam a presença de um fator, exceto pelo resultado da análise paralela no Estudo 1, que sugeriu a presença de dois fatores. Considerar essa estrutura bifatorial, além de rejeitar achados que têm sido congruentes na literatura, significa abrir mão de uma estrutura que oferece explicação mais parcimoniosa da fadiga. De fato, o Estudo 2 teve o papel de testar novamente a estrutura da escala com profissionais da saúde, corroborando, por meio de todos os critérios, a solução unifatorial. Destacase também que os resultados encontrados nos dois estudos quanto ao percentual de variância explicada, os valores do alfa de Cronbach, do $H$ de Löevinger e do $R$ ho de Mokken foram próximos aos encontrados no estudo original da construção da escala (Michielsen et al., 2004b).

Outro aspecto que merece ser discutido é a baixa carga fatorial do item 3 no Estudo 1 . Além de não ser adequado, decidiu-se por não eliminar esse item na versão final da escala, uma vez que, no Estudo 2, ele obteve carga fatorial satisfatória, e o valor do alfa de Cronbach não aumentaria caso ele fosse excluído. Esse item também apresentou valor de escalabilidade $(H s)$ abaixo do ideal nos dois estudos, mas os resultados mostraram que os índices de escalabilidade geral $(H$ e $R h o)$ da escala ficaram acima do ideal em ambos os estudos. Por fim, o coeficiente de congruência entre as soluções fatoriais dos Estudos 1 e 2 corrobora que a solução unifatorial é adequada à representação da fadiga.

Por fim, em termos de futuros estudos, é necessário realizar pesquisas com outros profissionais, o que permitirá conhecer a influência da fadiga em diferentes áreas laborais, analisando, por exemplo, se existe alguma ocupação profissional que proporcione mais fadiga do que outra. Também é recomendado comprovar a invariância fatorial dessa medida de fadiga, avaliando os parâmetros de saturação e erro de medida, por exemplo. Do mesmo modo, é importante checar a variação dos achados a partir de diferentes matrizes de entrada e métodos de estimação, assim como conhecer a relação desse construto com outros referidos na literatura, como a Síndrome de Burnout. No entanto, esse aspecto poderá facilitar o delineamento de ações interventivas que tenham em conta seus efeitos diversos.

\section{REFERÊNCIAS}

Andrea, H., Kant, I. J., Beurskens, A. J., Metsemakers, J. F., \& Van Schayck, C. P. (2003). Associations between fatigue attributions and fatigue, health, and psychosocial work characteristics: A study among employees visiting a physician with fatigue. Occupational and Environmental Medicine, 60(Suppl 1), 99-104. doi: 10.1136/oem.60.suppl_1.i99

Cella, M., \& Chalder, T. (2010). Measuring fatigue in clinical and community settings. Journal of Psychosomatic Research, 69(1), 17-22. doi:10.1016/j.jpsychores.2009.10.007

Chalder, T., Berelowitz, G., Pawlikowska, T., Watts, L., Wessely, S., Wright, D., \& Wallace, E. P. (1993). Development of a fatigue scale. Journal of Psychosomatic Research, 37(2), 147-153. doi: 10.1016/0022-3999(93)90081-P

Dagnelie, P. C., Pijls-Johannesma, M. C. G., Pijpe, A., Boumans, B. J. E., Skrabanja, A. T. P., Lambin, P., \& Kempen, G. I. J. M. (2006). Psychometric properties of the revised Piper Fatigue Scale in Dutch cancer patients were satisfactory. Journal of Clinical Epidemiology, 59(6), 642-649. doi: 10.1016/j.jclinepi.2005.09.015 
De Kleijn, W. P. E., Elfferich, M. D. P., \& De Vries, J. (2009). Fatigue in sarcoidosis: American versus Dutch patients. Sarcoidosis Vasculitis and Diffuse Lung Disease, 26(2), 92-97.

De Vries, J., Michielsen, H. J., \& Van Heck, G. L. (2003). Assessment of fatigue among working people: A comparison of six questionnaires. Occupational and Environmental Medicine, 60(Suppl 1), 10-15. doi: 10.1136/oem.60.suppl_1.i10

De Vries, J., Michielsen, H., Van Heck, G. L., \& Drent, M. (2004). Measuring fatigue in sarcoidosis: The Fatigue Assessment Scale (FAS). British Journal of Health Psychology, 9(3), 279-291. doi: 10.1348/1359107041557048

De Vries, J., Van der Steeg, A. F., \& Roukema, J. A. (2009). Determinants of fatigue 6 and 12 months after surgery in women with early-stage breast cancer: A comparison with women with benign breast problems. Journal of Psychosomatic Research, 66(6), 495-502. doi: 10.1016/j.jpsychores.2008.11.008

De Vries, J., Van der Steeg, A. F., \& Roukema, J. A. (2010). Psychometric properties of the Fatigue Assessment Scale (FAS) in women with breast problems. International Journal of Clinical and Health Psychology, 10(1), 125-139.

Elbers, R. G., Rietberg, M. B., Van Wegen, E. E., Verhoef, J., Kramer, S. F., Terwee, C. B., \& Kwakkel, G. (2012). Selfreport fatigue questionnaires in multiple sclerosis, Parkinson's disease and stroke: A systematic review of measurement properties. Quality of Life Research, 21(6), 925-944. doi: 10.1007/s11136-011-0009-2

Fritschi, C., \& Quinn, L. (2010). Fatigue in patients with diabetes: A review. Journal of Psychosomatic Research, 69(1), 33-41. doi: 10.1016/j.jpsychores.2010.01.021

Giallo, R., Wade, C., Cooklin, A., \& Rose, N. (2011). Assessment of maternal fatigue and depression in the postpartum period: Support for two separate constructs. Journal of Reproductive and Infant Psychology, 29(1), 69-80. doi: $10.1080 / 02646838.2010 .513050$

Hinz, A., Fleischer, M., Brähler, E., Wirtz, H., \& Bosse-Henck, A. (2011). Fatigue in patients with sarcoidosis, compared with the general population. General Hospital Psychiatry, 33(5), 462-468. doi: 10.1016/j.genhosppsych.2011.05.009

Janssen, N., Kant, I. J., Swaen, G. M. H., Janssen, P. P. M., \& Schröer, C. A. P. (2003). Fatigue as a predictor of sickness absence: Results from the Maastricht cohort study on fatigue at work. Occupational and Environmental Medicine, 60(Suppl 1), 71-76. doi: 10.1136/oem.60.suppl_1.i71

Kant, I. J., Bültmann, U., Schröer, K. A. P., Beurskens, A. J. H. M., Van Amelsvoort, L. G. P. M., \& Swaen, G. M. H. (2003). An epidemiological approach to study fatigue in the working population: The Maastricht cohort study. Occupational and Environmental Medicine, 60(Suppl 1), 32-39. doi: 10.1136/oem.60.suppl_1.i32

Krupp, L. B., LaRocca, N. G., Muir-Nash, J., \& Steinberg, A. D. (1989). The fatigue severity scale. Application to patients with multiple sclerosis and systemic lupus erythematosus. Archives of Neurology, 46(10), 1121-1123. doi: 10.1001/ archneur.1989.00520460115022

Lee, K. A., Hicks, G., \& Ninomurcia G. (1991). Validity and reliability of a scale to assess fatigue. Psychiatry Research, 36(3), 291298. doi: 10.1016/0165-1781(91)90027-M

Linsey, M., Barker, L. M., \& Nussbaum, M. A. (2011). Fatigue, performance and the work environment: A survey of registered nurses. Journal of Advanced Nursing, 67(6), 1370-1382. doi: 10.1111/j.1365-2648.2010.05597.x

Michielsen, H. J., De Vries, J., \& Van Heck, G. L. (2003). Psychometric qualities of a brief self-rated fatigue measure: The Fatigue Assessment Scale. Journal of Psychosomatic Research, 54(4), 345-352. doi: 10.1016/S0022-3999(02)00392-6

Michielsen, H. J., Willemsen, T. M., Croon, M. A., De Vries, J., \& Van Heck, G. L. (2004a). Determinants of general fatigue and emotional exhaustion: A prospective study. Psychology and Health, 19(2), 223-235. doi: 10.1080/08870440310001627135

Michielsen, H. J., De Vries, J., Van Heck, G. L., Van de Vijver, F. J. R., \& Sijtsma, K. (2004b). Examination of the dimensionality of fatigue: The construction of the Fatigue Assessment Scale (FAS). European Journal of Psychological Assessment, 20(1) 39-48. doi: http://dx.doi.org/10.1027/1015-5759.20.1.39

Michielsen, H. J., De Vries, J., Drent, M., \& Peros-Golubicic, T. (2005). Psychometric qualities of the Fatigue Assessment Scale in Croatian sarcoidosis patients. Sarcoidosis Vasculitis and Diffuse Lung Diseases, 22(2), 133-138.

Michielsen, H. J., Peros-Golubicic, T., Drent, M., \& De Vries, J. (2006). Relationship between symptoms and quality of life in a sarcoidosis population. Respiration, 548, 1-5. doi: 10.1159/000092670

Molenaar, I.W., \& Sijtsma, K. (2000). User's manual MSP5 for Windows. Groningen: IEC ProGAMM.

Moore, R., Gupta, P., \& Duval Neto, G. F. (2013). Fadiga ocupacional: Impacto na saúde do anestesiologista e a segurança dos pacientes cirúrgicos: Nós, como anestesiologistas estamos frequentemente trabalhando em um ambiente estressante. Você discorda disso? Revista Brasileira de Anestesiologia, 63(2), 167-169.

Mota, D. D. C. F., Pimenta, C. A. M., \& Caponero, R. (2012). Fadiga em pacientes com câncer colorretal: Prevalência e fatores associados. Revista Latino America de Enfermagem, 20(3), 1-9. 
Oliveira, G. F., Gouveia, V. V., Peixoto, G. P., \& Soares, M. A. L. (2010). Análise fatorial da escala de avaliação da fadiga em uma amostra de universitários de instituição pública. ID on line Revista de Psicologia, 4(11), 51-60.

Oliveira, J. R. S., Viganó, M. G., Lunardelli, M. C. F., Canêo, L. C., \& Goulart, E. (2010). Fadiga no trabalho: Como o psicólogo pode atuar? Psicologia em Estudo (Maringá), 15(3), 633-638.

Okuyama, T., Akechi, T., Kugaya, A., Okamura, H., Shima, Y., Maruguchi, M., Hosaka, T., \& Uchitomi, Y. (2000). Development and validation of the Cancer Fatigue Scale: A brief, three-dimensional, self-rating Scale for Assessment of Fatigue in cancer patients. Journal of Pain and Symptom Management, 19(1), 5-14. doi: 10.1016/S0885-3924(99)00138-4

Owens, J. A. (2012). Sleep loss and fatigue in healthcare professionals. The Journal of Perinatal \& Neonatal Nursing, 21(2), 92100. doi: http://dx.doi.org/10.1097/01.JPN.0000270624.64584.9d

Team, R. C. (2012). R: A language and environment for statistical computing. Recuperado de http://www.R-project.org/

Reynolds, C. R., \& Ramsey, M. C. (2003). Bias in psychological assessment: An empirical review and recommendations. In J. R. Graham \& J. A. Naglieri (Eds.), Handbook of psychology: Assessment psychology (Vol. 10, pp. 67-93). New York: John Wiley.

Sanches, K. C., \& Cardoso, K. G. (2012). Estudo da fadiga e qualidade de vida nos pacientes com doença de Parkinson. Journal of the Health Sciences Institute, 30(4), 391-394.

Schwartz, J. E., Janford, L., \& Krupp, L. B. (1993). The assessment of fatigue: A new measurement. Journal of Psychosomatic Researches, 37(7), 753-762. doi: 10.1016/0022-3999(93)90104-N

Shahida, A., Shena, J., \& Shapiro, C. M. (2010). Measurements of sleepiness and fatigue. Journal of Psychosomatic Research, 69(1), 81-89. doi: 10.1016/j.jpsychores.2010.04.001

Shapiro, C. M., Flanigan, M., Fleming, J. A., Morehouse, R., Moscovitch, A., Plamondon, J., ... Devins, G. M. (2002). Development of an adjective checklist to measure five FACES of fatigue and sleepiness. Data from a national survey of insomniacs. Journal of Psychosomatic Research, 52(6), 467-473. doi: 10.1016/S0022-3999(02)00407-5

Smets, E. M. A., Garssen, B., Bonk, B., \& de Haes, J. C. J. M. (1995). The Multidimensional Fatigue Inventory (MFI): Psychometric qualities of an instrument to assess fatigue. Journal of Psychosomatic Research, 39(3), 315-325. doi: 10.1016/0022-3999(94)00125-O

Smith, O. R., Michielsen, H. J., Pelle, A. J., Schiffer, A. A., Winter, J. B., \& Denollet, J. (2007). Symptoms of fatigue in chronic heart failure patients: Clinical and psychological predictors. European Journal of Heart Failure, 9(9), 922-927. doi: 10.1016/j. ejheart.2007.05.016

Tabachnick, B. G., \& Fidell, L. S. (2013). Using Multivariate Statistics (6a ed.). Boston, MA: Allyn and Bacon.

Van Dijk, F. J., \& Swaen, G. M. (2003). Fatigue at work. Occupational and Environmental Medicine, 60(Suppl 1), 1-3. doi:10.1136/ oem.60.suppl_1.i1

Vasconcelos, S. P., Fischer, F. M., Reis, A. O. A., \& Moreno, C. R. C. (2011). Factors associated with work ability and perception of fatigue among nursing personnel from Amazonia. Revista Brasileira de Epidemiologia, 14(4), 688-697. doi: http://dx.doi. org/10.1590/S1415-790X2011000400015

Yang, C. M., \& Wu, C. H. (2005). The Situational Fatigue Scale: A different approach to measuring fatigue. Quality of Life Research, 14(5), 1357-1362. doi: 10.1007/s11136-004-5680-0

Wilson, K. (2013). Social work under pressure: How to overcome stress, fatigue and burnout in the workplace. Practice: Social Work in Action, 25(2), 145-146. doi: 10.1080/09503153.2013.780751 